\title{
Interpretation of the Representational Meaning of Movie Posters from the Perspective of Multimodal Discourse Analysis
}

\author{
Yunru Chen \\ School of Foreign Languages \\ Wuhan Textile University \\ Wuhan, China \\ yunru-chen@qq.com
}

\author{
Xiaofang Gao \\ School of Foreign Languages \\ Central China Normal University \\ Wuhan, China \\ gxfang@mail.ccnu.edu.cn
}

\begin{abstract}
The advancement of modern information technology is challenging the dominant role of language in mass media and communication. The study of discourse analysis goes beyond language itself by incorporating other semiotic resources such as image, sound and animation into its scope. Considering language as a social semiotic and meaning potential, SystemicFunctional Linguistics provides theoretical foundation for multimodal discourse analysis (MDA). As pioneers, Kress and van Leeuwen proposed the grammar of visual design to interpret visual composition from representational meaning, interactive meaning and compositional meaning. With an interpretative approach, this paper intends to have an insightful discussion about how semiotic tools function in realizing the representational meaning of movie posters. The present study may not only enrich the application study of MDA, but also fill in a gap in discourse analysis of movie posters.
\end{abstract}

Keywords-multimodal discourse analysis; representational meaning; Systemic-Functional Linguistics; movie posters

\section{INTRODUCTION}

With the co-operation of two or more semiotic modes of communication including language, image, video, and sound, multimodal discourse creates an integrative meaning which makes the traditional discourse analysis restricted to language itself far from comprehensive and exhaustive. SystemicFunctional Linguistics developed by Halliday provides an effective theoretical tool for MDA. In light of the grammar of visual designs proposed by Kress \& van Leeuwen, we will have a comprehensive illustration for representational meaning by analyzing that of seven movie posters with distinctive features.

\section{LITERATURE REVIEW}

\section{A. Overview of Systemic-Functional MDA}

Systemic-Functional Linguistics is an approach to linguistics that considers language as a social semiotic system (Halliday 1978). Halliday supposes that language is a system network and the grammatical or other features of language are described as sets of choices. He also holds that all languages involve three meta-functions which come into being simultaneously: ideational function, interpersonal function and textual function (Halliday 1994). Although originally conceived for the study of language, this theory is widely used to analyze other semiotic resources. As the pioneering work, Kress and van Leeuwen's Reading Images (Kress \& van Leeuwen 1990) becomes the authority in interpreting visual compositions. They propose that the semiotic tools in visual communication are delicately represented to realize some particular functions, namely, representational meaning, interactive meaning and compositional meaning. In china, the study of MDA has been proceeding apace. Li Zhanzi (2003) firstly brings in the grammar of visual design proposed by Kress and van Leeuwen. Zhu Yongsheng (2007) articulates the definition of multimodality, the theoretical basis, methodology, and significance of MDA. Hu Zhuanglin points out the significance of enhancing people's general comprehension and identification on multimodal discourse. Zhang Delu (2009) deploys Systemic-Functional Linguistics to propose a synthetic theoretical framework for MDA. Based on their introductive work, the theory of MDA is beginning to be applied to different genres by Chinese scholars.

\section{B. Overview of discourse analysis of movie posters}

Movie poster, a form of visual communication, is designed to make an impression and entice movie goers. Nowadays, as the aesthetic and collection value are being paid more and more attention to, the study concerning how to give comprehensive analyses of movie posters is significant. However, up to the present, analyses of movie posters concentrate either on text only, or on aesthetic design, but very few analyses of movie posters are conducted to study the meaning-makings of all major modalities and of semiotic tools simultaneously.

\section{METHODS}

\section{A. Methods}

In discussion, an interpretative approach is adopted. Such an approach foregrounds depth over breadth and enables insightful analyses of chosen movie posters. 


\section{B. Data Selection}

Seven movie posters are chosen as the data for discussion for the following reasons: firstly, the movies introduced by these posters are with good popularity in public. Secondly, since each of the first six posters is an archetype of certain dimension of representational meaning, the discussion with focuses on their unique visual designs could illustrate how semiotic tools functions in the relevant processes mostlikely. Thirdly, the last poster touches upon almost every dimension of representational meaning, so the analysis of it could be a model of systemic and comprehensive interpretation of representational meaning of a movie poster.

\section{RESULTS}

Firstly, every dimension of representational meaning is illustrated adequately by examples of six movie posters with distinctive features. Secondly, a systemic and comprehensive explanation is given on how semiotic tools function jointly in realizing representational meaning of a movie poster. Thirdly, it is proved that the representational meaning reveals the internal relations of the represented visual elements of movie posters.

\section{DISCUSSION}

\section{A. RepresentationalMeaning in Movie Posters}

According to Kress \& van Leeuwen (1996:45), the representational meaning refers to how semiotic systems exhibit objects and relations between them outside the representational system or in the context of a culture. It is related to the internal relations between the represented participants, things, the action they perform as well as the setting of the circumstance. By the way, participants mentioned in the following discussion are divided into two kinds, namely, represented participants (people, things and places depicted in an image) and interactive participants (people who communicate with each other through images including image producers and viewers). The internal relations of visual images can be divided into two patterns---narrative process and conceptual process. The most important signal for distinguishing the two processes is whether there exists a vector.

\section{1) Narrative process}

Narrative process presents the development of actions and events, the processes of change and impermanent spatial arrangement, and its hallmark is the existence of a vector. In pictures, vectors are usually created by an oblique line which is formed by the represented elements (Kress \& van Leeuwen, 1996:57). In most cases, a quite strong diagonal line is used in displaying the vector. In naturalistic pictures, diagonal lines can be shaped by bodies, limbs or tools while in abstract pictures by abstract graphic elements. According to the types of vector and the number and kind of participants involved, narrative process can be classified into three sub-processes: action process, reactional process, as well as speech and mental process.

Above all, in an action process, there are two kinds of participants: the actor and the goal. The actor is the represented participant which emanates the vector, or which itself, in part or in whole, forms the vector, while the goal is the represented participant at which the vector is aimed, so it is also the objective of the action. In the poster of Schindler' List (see Fig.A1 in Appendix), the locus of attention is an adult's hand and a kid's hand holding together. The direction of their arms which forms the diagonal of this poster creates a vector and illustrates an action process. In this reciprocal action, the two represented participants are both the actor in one and the goal in another one. For such a movie as an epic drama of holocaust survivors in World War II and the man who unexpectedly came to be the saviour, this action process with remarkable humanitarian feelings plays a decisive role in giving a visual interpretation to the theme of movie---redemption.

In the second place, when the vector is formed by the eye line of the represented participants, the process is named reactional (Kress \& van Leeuwen 1996:64). In the poster of Mei Lanfang (see Fig.A2 in Appendix), there are two represented participants, a man and a woman, standing away from each other with no body contact but at the same time, looking at each other intently. Their eye line forms a vector which creates a reactional process, in which the two represented participants both serve as the reactors and the phenomena interchangeably.

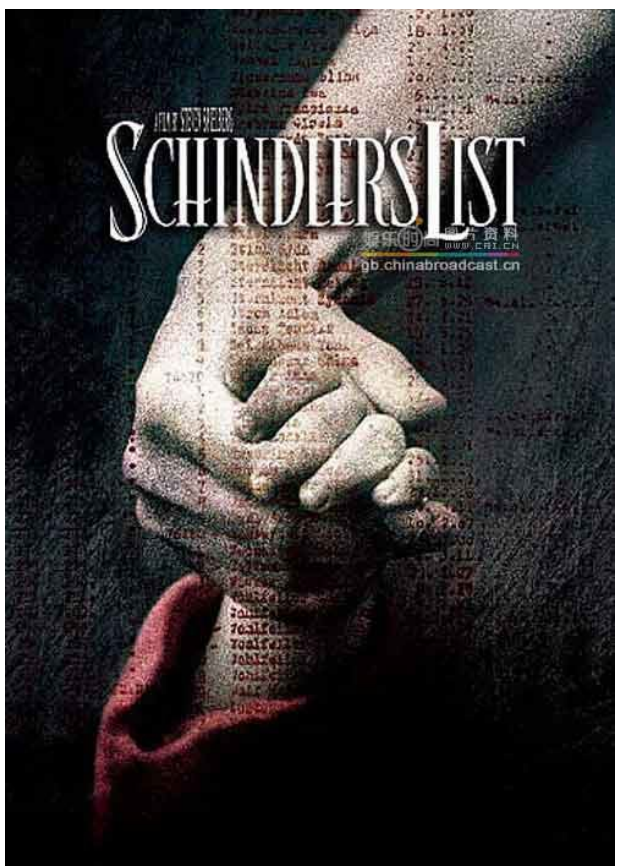

Fig. A1. Poster of Schindler' List 


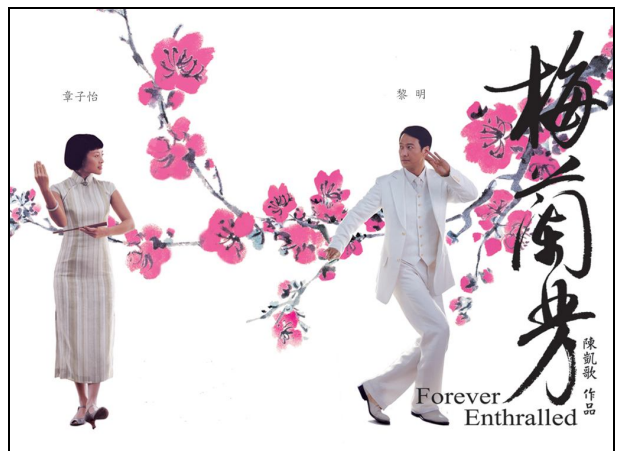

Fig. A2. Poster of Forever Enthralled

Thirdly, speech process and mental process typically refers to oblique protrusions of thought balloons or dialogue balloons used to connect a human being (or an animal) with his or her inner mental process or speech in comic strips. The participant who emanates the balloons is called sensor (in the case of a thought balloon) or speaker (in the case of the dialogue balloon).

At last, some minor represented participants, which are not related to the main participants by means of vectors, offer some basic information of an event such as the time, the place and the cause. These participants are termed as circumstance of setting, circumstance of means or circumstance of accompaniment. In the poster of The Queen (see Fig.A3 in Appendix), the Queen presented in the foreground is the main participant while a framed photo of huge size placed in the background is the secondary participant. Surprisingly, the main participant here only takes up less than $1 / 2$ of the frame; in contrast, the secondary participant takes up almost $1 / 2$ of the frame. The researcher thinks this design is unusual but justifiable. In fact, this movie is not a biography to recall the whole life of Queen Elizabeth II up to the present but an imagery story about the Queen based on a series of real happenings associated with the unexpected death of Princess Diana. Therefore, the framed photo in the background serves as circumstance of setting to provide important information about the background of the story. The young lady in the framed photo must be the Princess Diana, even though viewers cannot see the whole face. The color of the photo is made black and white to indicate the passing away of Princess Diana.

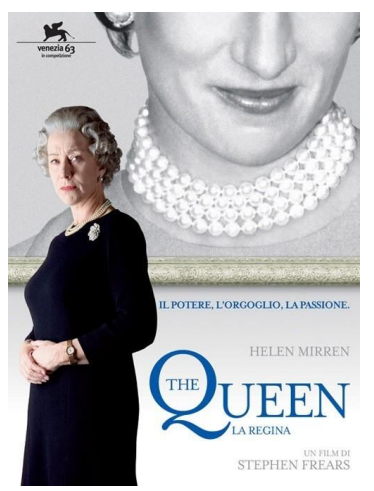

Fig. A3. Poster of The Queen

\section{2) Conceptual process}

In contrast to the dynamic nature of narrative process, conceptual process is more stable and more general, and it represents participants in terms of types, structure and meaning. Generally speaking, Conceptual processes can be classified into three sub-processes: classificational process, analytical process and symbolic process.

Firstly of all, in classificational process, the represented participants are related to each other in terms of taxonomy, among which several participants play the part of subordinates while at least one other acts as the superordinate. In an overt taxonomy, the superordinate is represented in the image overtly. This kind of classification process usually takes the form of diagram or tree structure. However, in a covert taxonomy the superordinate is either only hidden in the accompanying text, or indicated in the resemblance between the subordinates. In the realization of covert taxonomy, a symmetrical composition is crucial in visually realizing the proposed equivalence between the subordinates. Generally speaking, the subordinates are of the same size and placed at equal distance from each other. The Poster of Sen7en (see Fig.A4 in Appendix) is an archetype of covert taxonomy. Against the dark background, the subordinates, seven white-framed pictures of the same size, are almost positioned at symmetrical composition with equal distance from each other, thus the proposed equivalence between the subordinates is visually realized. The most conspicuous elements are seven scarlet words on the seven pictures respectively: Greed, Pride, Sloth, Gluttony, Envy, Lust and Wrath. These words stand for the crimes which are known as the Seven Deadly Sins. Therefore, in this covert taxonomy, the superordinate is actually indicated by the tagline at the top of the poster: "Seven deadly sins, Seven ways to die." Through that classificational process, the poster conveys abundant information about the synopsis of the movie and brings viewers great attraction and inspiration. Some key words may come into the viewers' mind, like a series of bizarre murders and seven Catholic death penalties.

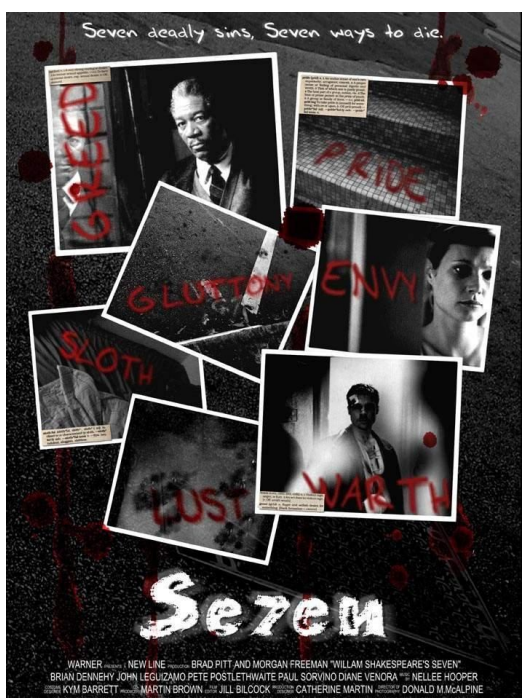

Fig A4. Poster A of Se7en 
Secondly, analytical process relates represented participants within a part-whole structure. There are two kinds of participants here: carrier which acts as the whole and possessive attributes as the parts. In another poster of Sen $7 e n$ (see Fig.A5 in Appendix), the most conspicuously represented participant is the Fig. "7", which takes up almost the whole of the frame. To decide a right angle of interpreting the " 7 " is the sticking point. Above all, a careful observation is needed. Firstly, it can be easily found that the image of "7" is made up of seven pictures which seem to be unrelated. However, in scrutinizing these pictures, viewers can discover some words including "Envy", "Pride", "Gluttony" and "Greed". Secondly, a tagline "LET HE WHO IS WITHOUT SIN TRY TO SURVIVE" is placed above the "7". In the tagline, all letters are capitalized for emphasis and all the letters " $T$ " are transformed into the form of cross with an indication of Catholicism. All those clues sufficiently show that "7", a mysterious figure in western culture, is used here to stand for the Seven Deadly Sins-Greed, Pride, Sloth, Gluttony, Envy, Lust and Wrath. Since the seven pictures and the "7" are presented within a part-whole structure, it is not difficult to recognize the spatial analytical process, within which the "7" is the carrier referring to the Seven Deadly Sins, while the seven pictures are the possessive attributes of the "7", referring to the content of the Seven Deadly Sins. Form this spatial analytical process, viewers could know better the synopsis and keynote of the movie.

Thirdly, symbolic process is about what a represented participant means or symbolizes. The represented participant whose nature or significance is demonstrated in the symbolic relation is called the carrier, while the one who represents the meaning or identity itself is termed as the attribute. The success of the poster of American Beauty (see Fig.A6 in Appendix) lies in the embedded symbolic meaning which conveys the theme of the movie visually. In the symbolic attribute process, the carrier is the one whose belly is presented as the background of the poster, and the attribute is the vermeil rose which is placed in the foreground with sharp focus. Judging from the flat belly and the satin skin, viewers could speculate that the one is probably a sexy young girl, a beauty; while the rose that is to blossom suggests the same meaning of youth and sex appeal; Based on this quality they share, the rose is designed to symbolize the girl's voluptuousness and attractiveness. What's more, as the saying goes, every rose has its thorns. So the rose as the attribute indicates the pain along with the love for the girl at the same time. In addition, the rose turns to be the carrier with a deep and covert symbolic meaning in a symbolic suggestive process. Above all, American Beauty itself is the name of a famous kind of rose which is beautiful but of short florescence. Therefore, the rose also symbolizes American dream which is beautiful superficially but doomed to fall to pieces.

\section{B. Analysis of the Represented Meaning of the Poster of The Flowers of War}

In term of the poster of The Flowers of War (see Fig.A7 in Appendix), the narrative meaning is embedded in an action process in which a dead branch in the top left corner forms an obvious vector. This dead branch extending towards the center of the image somehow serves as a stress and guide in visual communication in that it evidently draws viewers' attention to the bloody butterfly which is not only the goal of this vector, but also the most significant visual element in this poster.
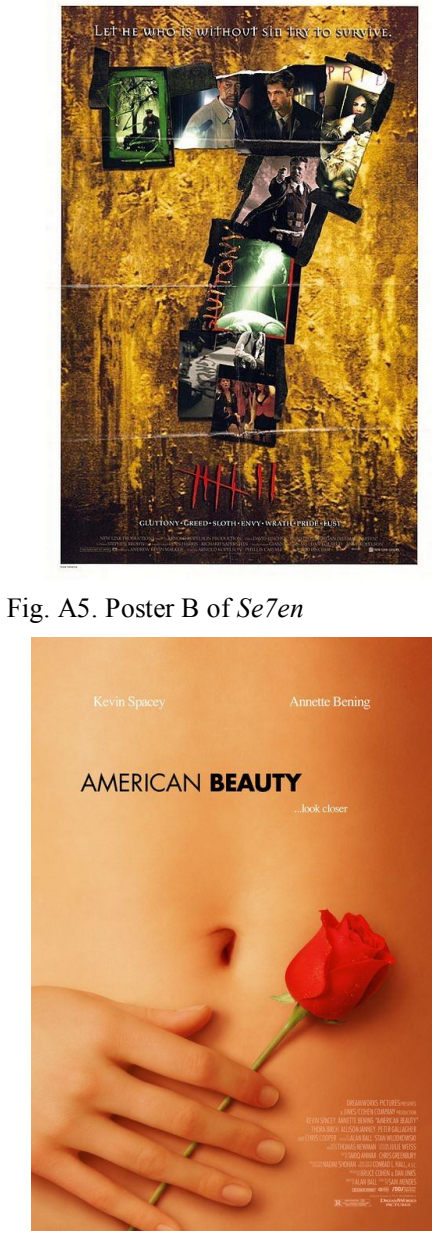

Fig. A6. Poster of Gone with the Wind

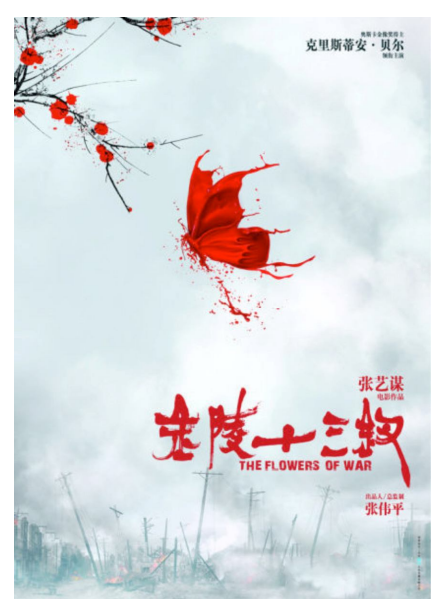

Fig. A7. Poster of The Flowers of War 
Moreover, the highlights of this poster lie in the conceptual process, especially the symbolic suggestive process. For one thing, from the choices of colors, all the important visual elements are presented in the color of blood-red which symbolizes death and killing and thus conveys the impression of an extremely bloody and brutal story to viewers. The connotation of blood red accords with the emotional tone of the movie background, a famous historical event ---Nanking Massacre. For another, from the choices of visual elements, the blood spots splashed on the dead branch are designed just like blooming red plum blossoms and thus endowed with multiple symbolic meanings: firstly, the English title "The Flowers of War" employs a figure of speech called metonymy. The vehicle is "flowers" and the tenor is women. The poster designers choose to illustrate the word "flowers" with the imageries of red plum blossoms which may share the same connotation and essence worldwide. Especially in Chinese culture, the imageries of plum blooms are the symbols of many virtues such as fortitude, bravery, determination, and indifference to fame and wealth. Just as a well-known Chinese poem writes "there are a few plum trees in the corner of garden; they bloom alone in the struggle against severe cold". Therefore, the choice of the imageries of red plum blossoms is well-grounded, and they just hint the lofty morals and sentiments of the leading characters; secondly, as an old Chinese saying goes, east or west, the plum blossoms in Jinling is best. Here "Jinling" is the former name of Nanking. Hence, the imageries of red plum blossoms related to "Jinling" in the Chinese title are suggesting the place of the story; thirdly, the blooming season of plum trees coincides with the story time (Nanking Massacre took place on the $13^{\text {th }}$ of December in 1937 and last for about 6 weeks), and the bitter cold in severe winter could be regarded as an indication of the violent and ruthless social environment of that day..

The last but not the least, it seems farfetched outwardly to design a bloody butterfly in symbolic suggestive process; however, it actually places an irreplaceable role in delivering the subject of this poster and also in stressing the theme of selfredemption in the movie. The metamorphosis from an ugly and awkward larva into a chrysalis and then into a beautiful and graceful butterfly symbolizes the transformation of the leading characters' human nature, or instance, from a greedy drunkard to a courageous and beneficent hero, from a gang of vulgar and selfish prostitutes to a group of honorable women volunteering to give up their lives to save the girl students, and from a lackey willing to sell the country for his own selfish ends to a valiant risking his own life to help the girl students to run for their lives. All those are true portraitures of the Chinese people's self-redemption from the suffering and sorrow at the time of national disaster. In addition, these is a household Chinese legend of a tragic love story named Butterfly Lovers, so the image of butterfly could also be taken as the emblem of the love between the hero and heroin which is beautiful but short-lived and ends abruptly with death.

\section{Conclusion}

Through discussion, it is proved that representational meaning is realized to reflect the internal relationship of represented participants in movie posters. Accurate interpretation of representational meaning of a poster is the prerequisite to appropriate prediction of the movie story, and is also indispensable to the sound understanding of the theme and keynote of a movie. The key to uncovering representational meaning is the recognition and analysis of every dimension of narrative process and conceptual process which may possibly exist in a poster.

\section{REFERENCES}

[1] Halliday, M. A. K. Language as social semiotic: The social interpretation of language and meaning [M]. London: Edward Arnold, 1978

[2] Halliday, M. A. K. An introduction to functional grammar [M]. London: Edward Arnold, 1994.

[3] Kress, G. \& van Leeuwen, T. Reading images [M]. Geelong: Daekin UP, 1990.

[4] Kress, G. \& van Leeuwen, T. Reading images: The grammar of visual design [M]. London: Routledge, 1996.

[5] Chen Yunru. A multimodal discourse analysis of movie posters [A]. Proceedings of $2^{\text {nd }}$ Annual International Conference on Language, Literature\& Linguistics, Singapore, 2013

[6] Chen Yunru. The interpretation of movie posters from the perspective of multimodal discourse analysis. [J]. GSTF Journal on Education, Vol.1, No.1, 2013, pp. 76-99

[7] $\mathrm{Hu}$ Zhuanglin..Multimodality in Social Semiotics [J]. Language Teaching and Linguistic Studies, 2007,(1): pp1-10 (in Chinese)

[8] Huang Guowen. Theory and Practice of Discoures Analysis[M].Shanghai: Shanghai Foreign Language Education Press, 2001

[9] Li Zhanzi. Social Semiotic Analysis of Multimodal Discourse [J]. Foreign Language Research, 2003, (5): pp. 1-4 (in Chinese)

[10] Zhang Delu. On a Synthetic Theoritical Framework for MDA [J]. Foreign Language Research, 2009, (1): pp. 24-30 (in Chinese)

[11] Zhu Yongsheng. Theory and Methodology of MDA [J]. Foreign Language Research, 2007, (5): pp. 82-86 (in Chinese) 